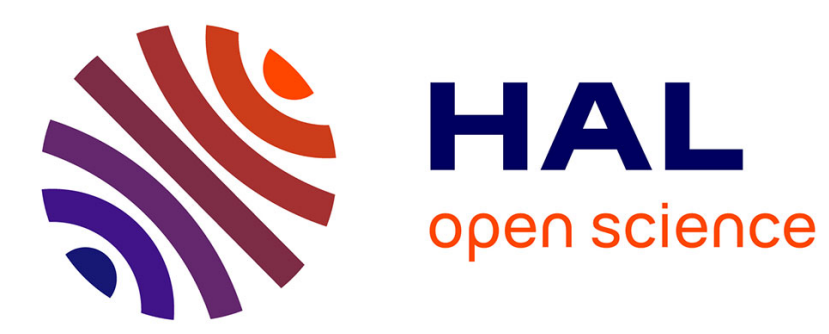

\title{
Presentation and improvement of an AFM-based system for the measurement of adhesion forces.
}

Micky Rakotondrabe, Patrick Rougeot

\section{To cite this version:}

Micky Rakotondrabe, Patrick Rougeot. Presentation and improvement of an AFM-based system for the measurement of adhesion forces.. The Fifth IEEE International Conference on Automation Science and Engineering, IEEE CASE’09., Aug 2009, Bangalore, India. pp.585-590. hal-00418769

\section{HAL Id: hal-00418769 \\ https://hal.science/hal-00418769}

Submitted on 21 Sep 2009

HAL is a multi-disciplinary open access archive for the deposit and dissemination of scientific research documents, whether they are published or not. The documents may come from teaching and research institutions in France or abroad, or from public or private research centers.
L'archive ouverte pluridisciplinaire HAL, est destinée au dépôt et à la diffusion de documents scientifiques de niveau recherche, publiés ou non, émanant des établissements d'enseignement et de recherche français ou étrangers, des laboratoires publics ou privés. 


\title{
Presentation and improvement of an AFM-based system for the measurement of adhesion forces
}

\author{
Micky Rakotondrabe, Member, IEEE, and Patrick Rougeot
}

\begin{abstract}
The aim of this paper is the presentation and improvement of an AFM-based system dedicated to measure adhesion forces.

Because an AFM-lever presents a high linearity and a high resolution, it can be used to characterize forces that appears between two micro-objects when their relative distance is small. In this paper, an AFM is used to evaluate the adhesion forces versus the distance. Especially, the pull-off and the Van Der Waals forces can be quantified. Unfortunately, the presence of the hysteresis on the piezotube distorts the measurement and makes the whole system imprecise. Hence, a Prandtl-Ishlinskii hysteresis compensator is introduced. To show the efficiency of the improved measurement system, experiments on different materials where performed.
\end{abstract}

\section{INTRODUCTION}

When two small objects, regularly less than one millimeter sizes, are interacted, forces that are negligible in classical sizes become important. These forces, called adhesion forces, are highly dependent on the surface of the microscopic objects and are paramount relative to the volume force (weight) [1]. Adhesion forces could be due to electrostatic, Van Der Waals or humidity of the media inside which the interaction happens [2].

In micromanipulation or microassembly, the achievement of the tasks are often compromised by the adhesion forces. A familiar example is the difficulty to release a micro-object during a robotic microassembly task (Fig. 1) (example in [3]). Due to the predominance of the adhesion forces relative to the weight, the microobject sticks on the manipulator when releasing it.

In order to account the adhesions forces during the design or in the control of systems dedicated to micromanipulation/microassembly, it is necessary to quantitatively or qualitatively have an évaluation of them. For that, theoretical formulation and analysis have been raised [4][5]. To validate the theory, the latter reference notably used an AFM-based measurement system to estimate the adhesions forces. While the experimental results well fit to the prediction, the measurement system only works with low value of adhesion forces (less than $30 n N)$. Indeed, the nonlinearity (hysteresis) of the piezotube were not taken into account and the accuracy of

FEMTO-st Institute,

UMR CNRS-6174 / UFC / ENSMM / UTBM

Automatic Control and Micro-Mechatronic Systems department (AS2M department)

25000 Besançon - France

mrakoton@femto-st.fr, patrick.rougeot@ens2m.fr

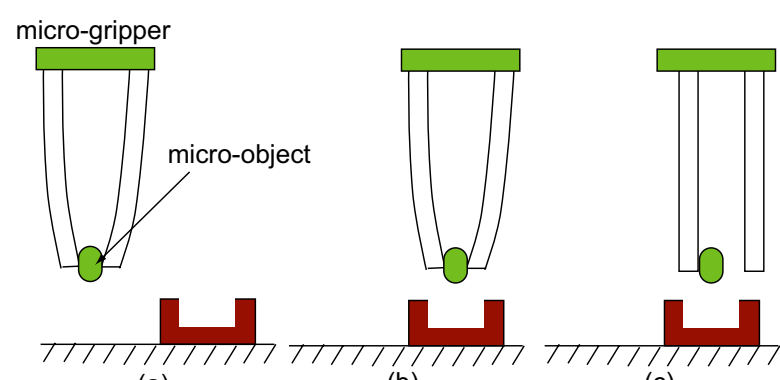

(a)

(b)

(c)

Fig. 1. Difficulty to release a micro-object during a microassembly task.

the measurement system is lost when working measuring forces with high amplitude.

In this paper, the measurement system is extended to high amplitude forces. The experiments are performed with values up to $400 n N$. To ensure the linearity of the system, and then the accuracy, the hysteresis is compensated. A Prandtl-Ishlinskii model is especially used due to its precision and embarkability.

The paper is organized as follow. First, the measurement system is presented. Afterwards, we detail the improvement of its accuracy by compensating the hysteresis. Finally, the experimental results with glass and gold substrates end the paper.

\section{Presentation of the force measurement SYSTEM}

The aim of the system is to permit the characterization of the adhesion forces (Van Der Waals forces, electrostatic force, capillary forces) between micro-objects. These adhesion forces appear when the distance between the two interacted objects are small or when they are in contact.

Because of the micro and nanometric range of the force to be characterized, the system is based on an AFMsystem. In fact, it provides a high resolution.

\section{A. Presentation of the system}

Fig. 2 pictures the principle of the micro-force measurement system:

- A substrate represents a micropart whose adhesion forces are to be characterized. The position of the substrate can be precisely adjusted using a motorized XYZ-table.

- An AFM-lever is used to characterize the adhesion forces. In fact, the characterized adhesions forces are 
the interaction force between the substrate and the tip of the AFM-lever.

- A piezoelectic tube actuator (piezotube) is used for the XYZ positioning of the AFM-lever. In this analysis, the piezotube only works on the Z-axis in order to perform an approach and a retract of the AFM-lever relative to the substrate.

- An optical sensor, based on a sensitive photodiodediode, measures the deflection $\delta_{l}$ of the AFM-lever.

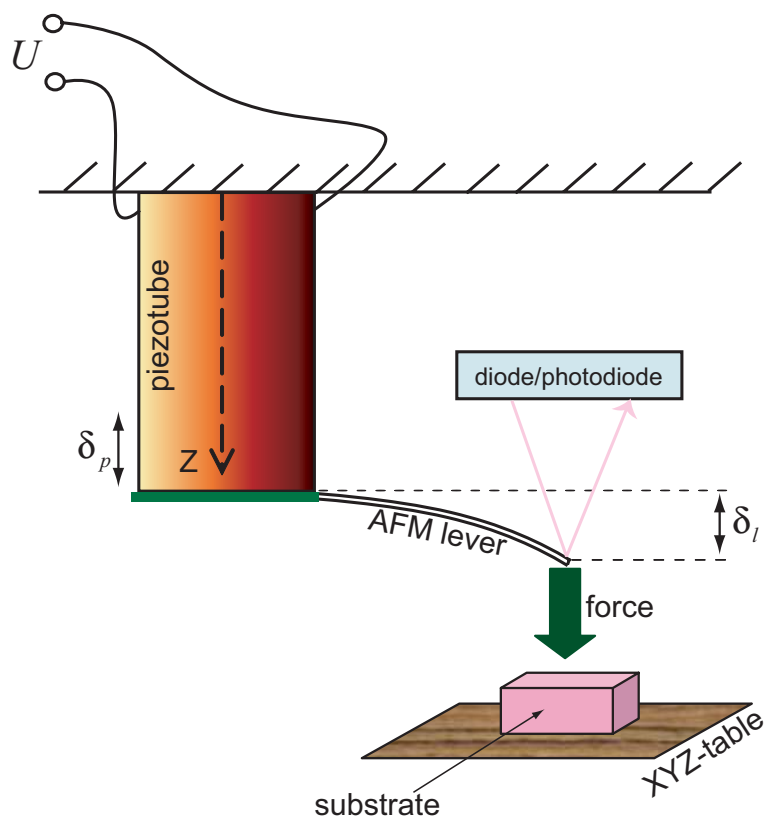

Fig. 2. Principle of the force measurement system.

A general view of the equipment is presented in Fig. 3. The used piezotube can perform $0 \leftrightarrow 4 \mu m$ of range in its $Z$-axis. The motorized XYZ-table is used to precisely align the substrate under the AFM-lever. Finally, a computer with a homemade software ( $\mathrm{C}$ code) gives the possibility to make automatic measurement cycles, to control the system and to read-out and save the measured signals from the sensor.

\section{B. Principle of the adhesion forces measurement}

Let $F$ designate the whole adhesions forces between the tip of the AFM-lever and the substrate. This force is given by:

$$
F=k_{A F M} \cdot \delta_{l}
$$

where $k_{A F M}$ is the stiffness of the AFM-lever. We consider that the force and the AFM-lever's deflection $\delta_{l}$ have the same positive direction.

To characterize the adhesion forces, we measure the force $F$ versus the relative distance between the AFMlever and the substrate. To perform that, the AFM-lever is approached up to the substrate until the contact and a preload force $F_{\max }$ are obtained. Afterwards, the AFMlever is moved away.

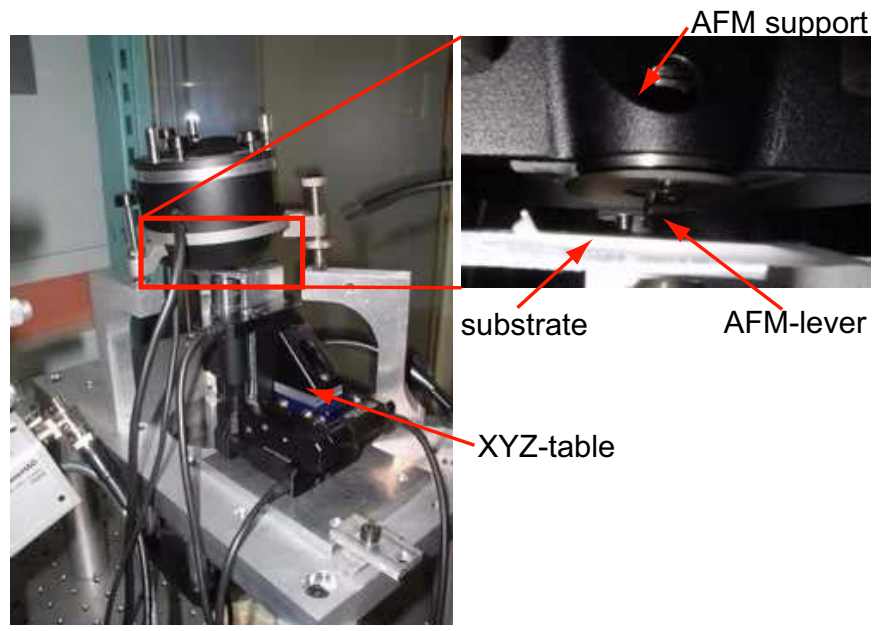

Fig. 3. Presentation of the setup.

Let the Fig. 4 present the principle of one measurement cycle, i.e. an approach-retract cycle. Because the relative distance between the AFM-lever and the substrate are not measurable, we use the expansion $\delta_{p}$ of the piezotube (see Fig. 2). The results, i.e. plots of $F$ versus $\delta_{p}$, permit analyzing the adhesion forces. We first suppose that the measurement of $\delta_{p}$ is available.

When a voltage excitation $U$ is applied to the piezotube, it extends. If the distance between the AFM-lever and the substrate are large enough, the interaction force between them is insignificant and the AFM-lever does not bend (Fig. 4-a). So, the measured force $F$ is equal to zero (Fig. 4-b).

If $\delta_{p}$ is continuously increased by increasing $U$, there will be a distance at which the interaction force is enough to attract the AFM-lever. Therefore, the latter start bending (Fig. 4-c). As the AFM-lever's deflection is negative, a negative interaction force $F$ is obtained (Fig. 4d). This interaction force include the Van Der Waals forces, the elctrostatic force and the capillary force. Their repartition strongly depends on different parameters: the ambient humidity, the surface characteristics of the objects, etc.

At a critical distance, the tip of the AFM-lever is suddenly pulled to stick on the substrate (Fig. 4-e). The corresponding force is called $F_{B}$ (Fig. 4-f).

If $\delta_{p}$ is still increased, the deflection $\delta_{l}$ of the AFMlever becomes first null and then positive (Fig. 4-g). Hence, the measured force $F$ ranges from $F_{B}$ (negative) to $F_{C}$ (positive) (Fig. 4 -h). The maximum value $F_{\max }=$ $F_{C}$ corresponds to the preload. The Van Der Waals forces are given by $F_{v d w}=F_{B}[5]$.

Now let us move away the AFM-lever from the substrate by decreasing $\delta_{p}$. The deflection $\delta_{l}$ becomes null and then negative (Fig. 4-i). As pictured in Fig. 4-j, the force ranges from $F_{C}>0$ up to a negative value.

When the force $F$ reaches a limit value $F_{D}<0$, the contact between the tip of the AFM-lever and the 
substrate is suddenly broken. As a result, the deflection $\delta_{l}$ (and therefore the intensity of $F$ ) brusquely decreases (Fig. 4-k, l). The force $F_{D}$ corresponds to the pull-off force [5].

From Fig. 4-1, the approach curve is defined by the following sequence:

$$
O \rightarrow A \rightarrow B \rightarrow C
$$

and the retract curve by:

$$
C \rightarrow D \rightarrow E \rightarrow O
$$

Considering that the behavior of the AFM-lever is linear, the slope of $C \rightarrow D$ should be equal to the slope of the $B \rightarrow C$. This slope should correspond to the stiffness $k_{A F M}$ of the AFM-lever. We have previously supposed that $\delta_{p}$ was measured. Nevetheless, one of the main limitations to the measurement and control in micro/and nano- systems is the lack of convenient and easily integrable sensors. This is why openloop control techniques are often used [6][7][8]. Notably, it is impossible in the presented system to introduce a sensor to measure $\delta_{p}$ as the lower part of the system is dedicated to the location of the XYZ-table and the substrate. Therefore, we will estimate the expansion of the piezotube from the applied voltage $U$. This part will be detailed in the next section.

\section{Estimation of THE EXPANSiON $\delta_{p}$ OF THE PIEZOTUBE}

Let us open loop control the piezotube. In the static mode, in order to obtain $\delta_{p}=\delta_{p}^{r e f}$, the gain of the feedforward controller should be $\frac{U}{\delta_{p}^{r e f}}=\frac{1}{d_{p}}$, where $d_{p}$ is a piezoelectric constant and $\delta_{p}^{r e f}$ is the reference input (Fig. 5-a). Therefore, we can use the available data $\delta_{p}^{r e f}$ instead the unavailable $\delta_{p}$ to perform the plots of Fig. 4. Unfortunately, the piezotube exhibits hysteresis effect and the $\delta_{p}^{r e f}$ differs from $\delta_{p}$. Then, to improve the accuracy, we use a feedforward controller that accounts the hysteresis (Fig. 5-b). We choose the Prandtl-Ishlinskii (PI) model and its compensator because its ease of implementation and low memory consumption make it very practical.

\section{A. Modeling the hysteresis $\Gamma(U)$ using the PI model}

Let $\Gamma(U)$ be the operator that models the static hysteresis of the piezotube. In the PI model, $\Gamma(U)$ is approximated by the sum of many elementary backlashes (Fig. 6-a). Each backlash is characterized by its bandwidth $b \omega_{i}$ and is weighted by a gain $w_{i}$ [9]. We have $b w_{i}=2 r_{i}$, where $r_{i}$ is the radius (or threshold) of the backlash. Fig. 6-b shows an example of hysteresis approximated by three elements [10].

From Fig. 6-b, the $k^{\text {th }}$ output can be formulated as follow:
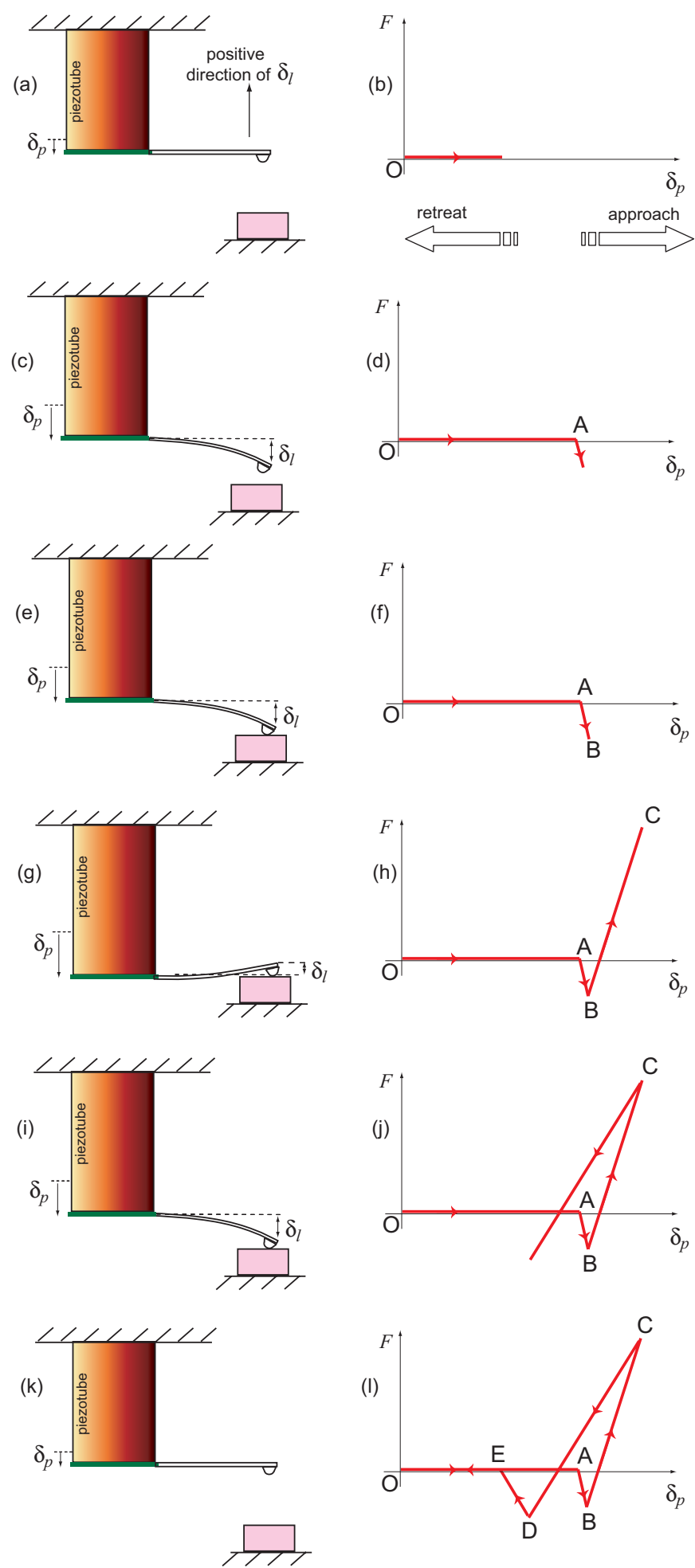

Fig. 4. Principle of the micro-forces measurement. 


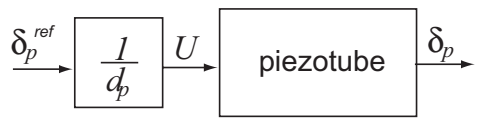

(a)

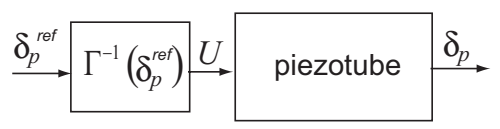

(b)

Fig. 5. Principle of the micro-forces measurement.
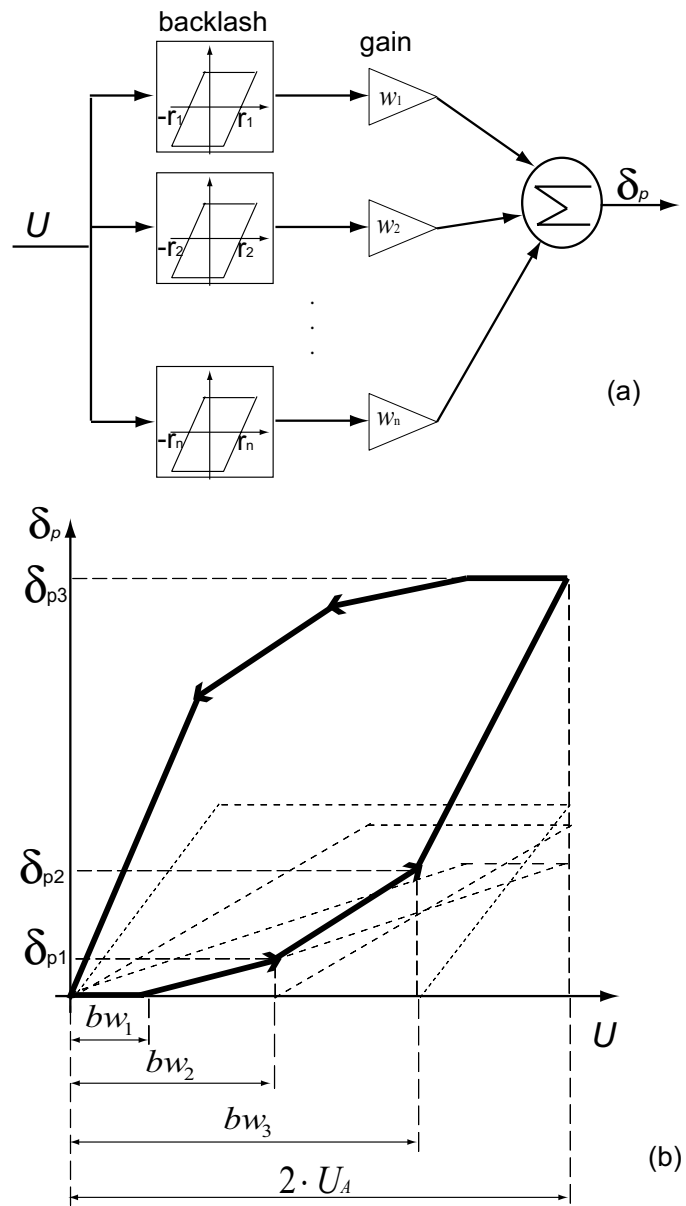

(b)

Fig. 6. Schemes showing the principle of the PI model.

$$
\delta_{p k}=\sum_{i=1}^{k}\left(b w_{k+1}-b w_{i}\right) \cdot w_{i}
$$

Hence, a tensorial formulation can be got:

$$
\left\{\delta_{p}\right\}=[A] \cdot\{w\}
$$

where $[A]$ is a triangular matrix constructed from the different bandwidth values.

To model and identify the hysteresis by the PI model, we use the following steps.
- Apply at least a half period of sine voltage $U$ to the piezocantilever. The amplitude of the corresponding output $\delta_{p}$ should cover the end use range.

- If the obtained hysteresis curve is not in the positive section of the $\left(U, \delta_{p}\right)$-plane, shift the curve.

- Define the number $n$ of the elementary backlashes.

- Split the input $U$ domain into $n+1$ uniform or nonuniform partitions. As example, Fig. 6-b pictures four partitions and presents an approximation of hysteresis with three backlashes. The bandwidth $b w_{i}$ and the output vector $\left\{\delta_{p}\right\}$ are easily obtained according to the Fig. 6-b.

- Construct the matrix $[A]$ from the bandwidth $b w_{i}$ using the (equ 4).

- Finally, compute the parameter $\{w\}$ using the following formula:

$$
\{w\}=[A]^{-1} \cdot\left\{\delta_{p}\right\}
$$

\section{B. Compensation of the hysteresis}

To compensate a hysteresis $\Gamma(U)$ that has been modelled with a PI model, another PI hysteresis model is put in cascade with it. Hence, the inverse model $\Gamma^{-1}\left(\delta_{p}^{r e f}\right)$ is also a sum of elementary backlashes each one characterized by a bandwidth $b w_{i}^{\prime}$, a threshold $r_{i}^{\prime}=\frac{b w_{i}^{\prime}}{2}$ and a weighting gain $w_{i}^{\prime}$.

The compensation principle is given in the Fig. 7: to obtain a linear input-output $\left(\delta_{p}^{r e f}, \delta\right)$ with a unit gain, the direct model curve $\left(U, \delta_{p}\right)$ and the inverse model curve $\left(\delta_{p}^{r e f}, U\right)$ should be symmetric themselves [11].

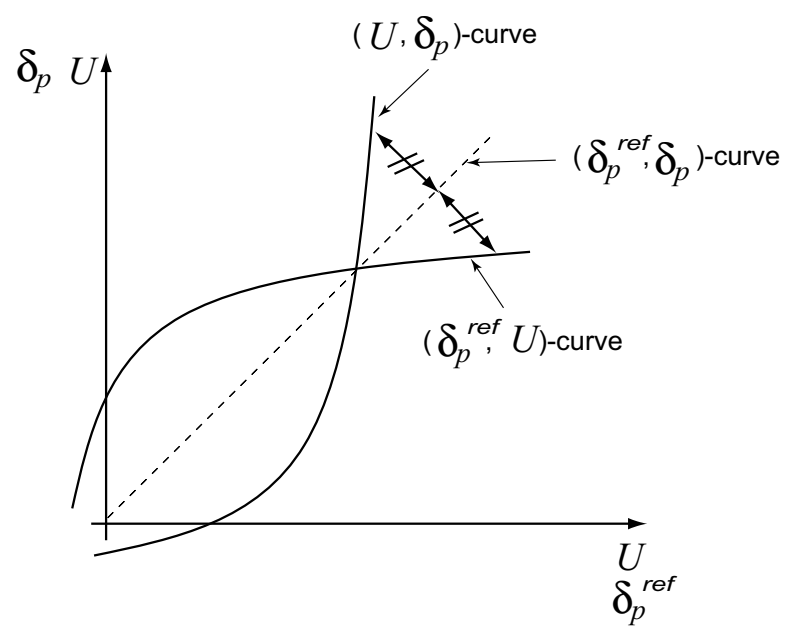

Fig. 7. Schemes showing the compensation of a hysteresis [11].

Using the principle pictured in Fig. 7, the parameters of the inverse model are computed as follow [11]:

$$
r_{k}^{\prime}=\sum_{j=1}^{k} w_{j} \cdot\left(r_{k}-r_{j}\right) \quad ; \quad k=1 \cdots n
$$

and 


$$
\begin{aligned}
& w_{1}^{\prime}=\frac{-1}{w_{1}} \\
& w_{k}^{\prime}=\frac{-w_{k}}{\left(w_{1}+\sum_{j=2}^{k} w_{j}\right) \cdot\left(w_{1}+\sum_{j=2}^{k-1} w_{j}\right)} \quad ; \quad k=2 \cdots n
\end{aligned}
$$

\section{Experimental results on the $\delta_{p}$ estimation}

In order to identify the $\Gamma(U)$ operator, a sine input $U$ has been applied to the piezotube. The frequency is chosen to be largely than the cutt-off frequency of the piezotube dynamic. The aim is to avoid the frequency effect on the shape of the hysteresis curve [12]. We choose $f=0.1 \mathrm{~Hz}$. On the other hand, we choose $n=15$ backlashes to model the hysteresis. If $n$ is too low the model accuracy is bad, but if $n$ is too high the model complexity is increased. Fig. 8 shows the experimental result and the simulation of the identified model $\Gamma(U)$.

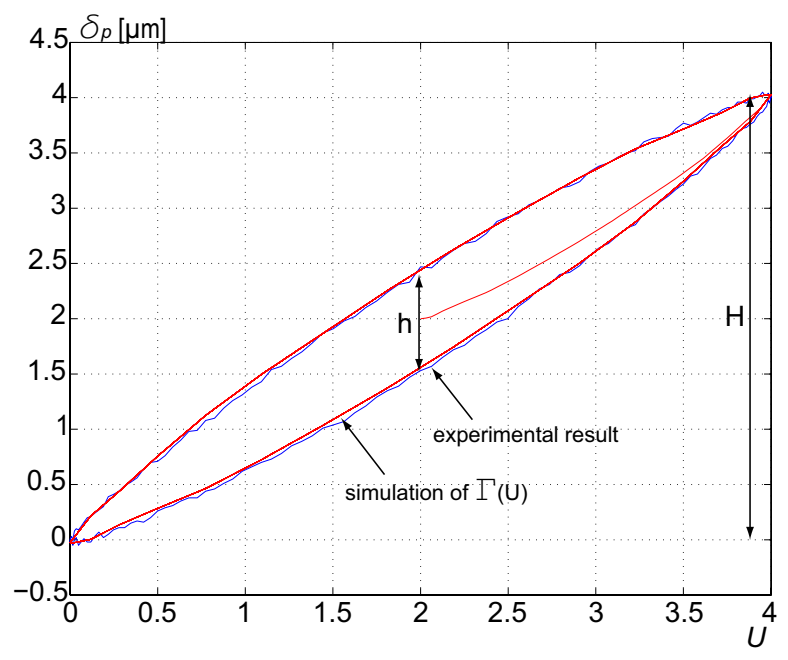

Fig. 8. Hysteresis curves: experimental result and identification result.

A compensator $\Gamma^{-1}\left(\delta_{p}^{r e f}\right)$ has been computed. To check its efficiency, a sine reference input $\delta_{p}^{r e f}$ with $3 \mu \mathrm{m}$ of amplitude is applied. Fig. 9 pictures the experimental result and points out that the hysteresis which was initially about $25 \%\left(=\frac{h}{H} \cdot 100 \%\right)$ is clearly deleted.

\section{Force measurement: experimental Results}

The first experiment concerns the analysis of adhesion forces between the AFM-lever and a glass substrate. To show the efficiency of the compensator, the experiment was performed on the system with and without hysteresis compensator. Tests with two preloads were carried out: $F_{C} \approx 200 n N$ and $F_{C} \approx 400 n N$. The results are pictured in Fig. 10-a for the former and in Fig. 10-b for the latter. They show that the pull-off force, equal to $F_{D} \approx 150 \mathrm{nN}$, is independant on the preload. On the other hand, the results clearly show that when the experiment is performed without the hysteresis compensator, the force evolution defined by the curve seems wrong. Indeed, during the

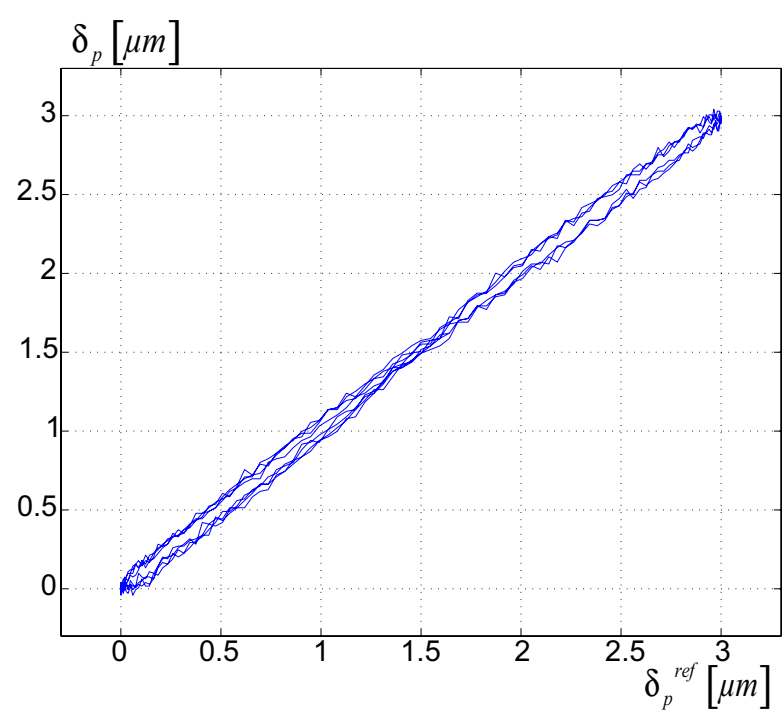

Fig. 9. Experimental result of the hysteresis compensation.

contact between the AFM-lever and the substrate, the stiffness of the AFM-lever can be experimentally estimated:

$$
k_{A F M}=\frac{\partial F}{\partial \delta_{p}}=\frac{\partial F}{\partial \delta_{l}}
$$

It seems that both the approach and the retract curves give wrong values. They should provides $k_{A F M}=$ $200\left[\frac{n N}{\mu m}\right]$ which corresponds to the stiffness of the used AFM-lever. As a conclusion, when the hysteresis is not compensated, the measured force is not coherent and cannot be used to evaluate the evolution of the adhesion forces. However, when the hysteresis is compensated, the approach and the retract curves well coincide.

The same experiment was performed between the AFM-lever and a gold substrate (Fig. 11). Here, the preload is chosen to be $F_{C} \approx 75 n N$ (Fig. 11-a) and then $F_{C} \approx 150 n N$ (Fig. 11-b). Similarly to the previous remarks, the results with the hysteresis compensator give coherent force evolution. Finally, the measured pull-off force is $F_{D} \approx 120 n N$ whatever the preload is.

\section{Conclusion}

This paper presents an AFM-based force measurement system and its improvement. The final objective is to have a system that can characterize the adhesion forces between the AFM-lever and a substrate. Because of the hysteresis characteristic of the piezotube, the measurement system presents an imprecision of the measured force. Hence, a hysteresis compensator has been computed and implemented. The Prandtl-Ishlinskii model has been chosen because of its accuracy and embarkability. Finally, experiments with two different objects (AFM-lever/glass and AFM-lever/gold) were performed in order to validate the efficiency of the improved measurement system. 

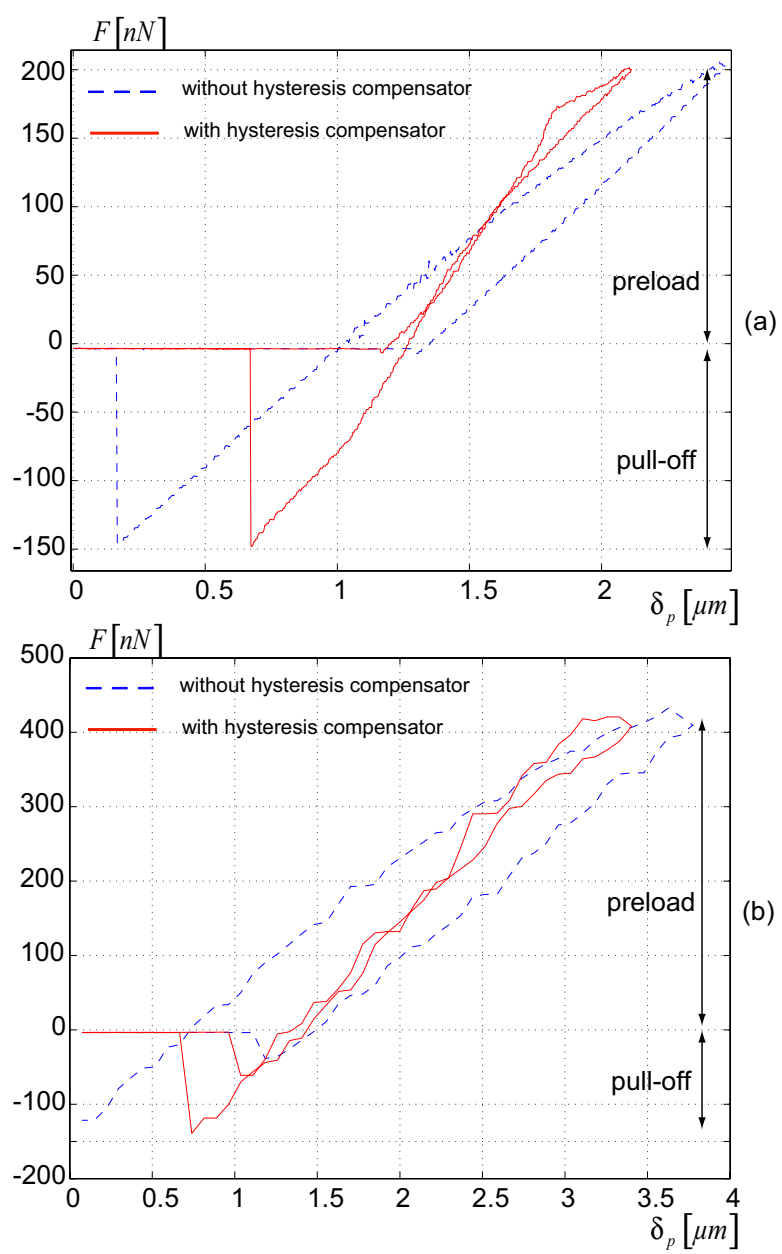

Fig. 10. Experimental result: measurement of the pull-off force between the AFM-lever and a glass substrate.

\section{ACKNOWLEDGMENT}

This work is supported by the French research project NANOROL (ANR NºPIROB07_184846).

\section{REFERENCES}

[1] R. Feynman, "Infinitesimal machinery", Journal of MicroElectroMechanical Systems, 2, No 1:4-14, 1993.

[2] R. S. Fearing, "Survey of sticking effects for micro parts handling", IEEE Internation Conference on Intelligent Robots and Systems, 1995.

[3] M. Weck, "Assembling hybrid microsystems", IAP Workshop - Advanced Mechatronic Systems, Lovain-La-Neuve, October 2003.

[4] J. Israelachvili, "Intermolecular and Surface Forces", Academic Press, 1991.

[5] M. Gauthier, S. Régnier, P. Rougeot and N. Chaillet, "Forces analysis for micromanipulations in dry and liquid media", Journal of MicroMechatronics, 3, pp.389-413, 2006.

[6] B. Mokaberi and A. A. G. Requicha, "Compensation of scanner creep and hysteresis for AFM nanomanipulation", IEEE Transactions on Automation Science and Engineering, pp.197 - 206, April, 2008.

[7] S. Devasia, E. E. Eleftheriou, R. Moheimani, "A survey of control issues in nanopositioning", IEEE Transactions on Control Systems Technology, Vol.15, $N^{o} 15$, pp.802-823, 2007.
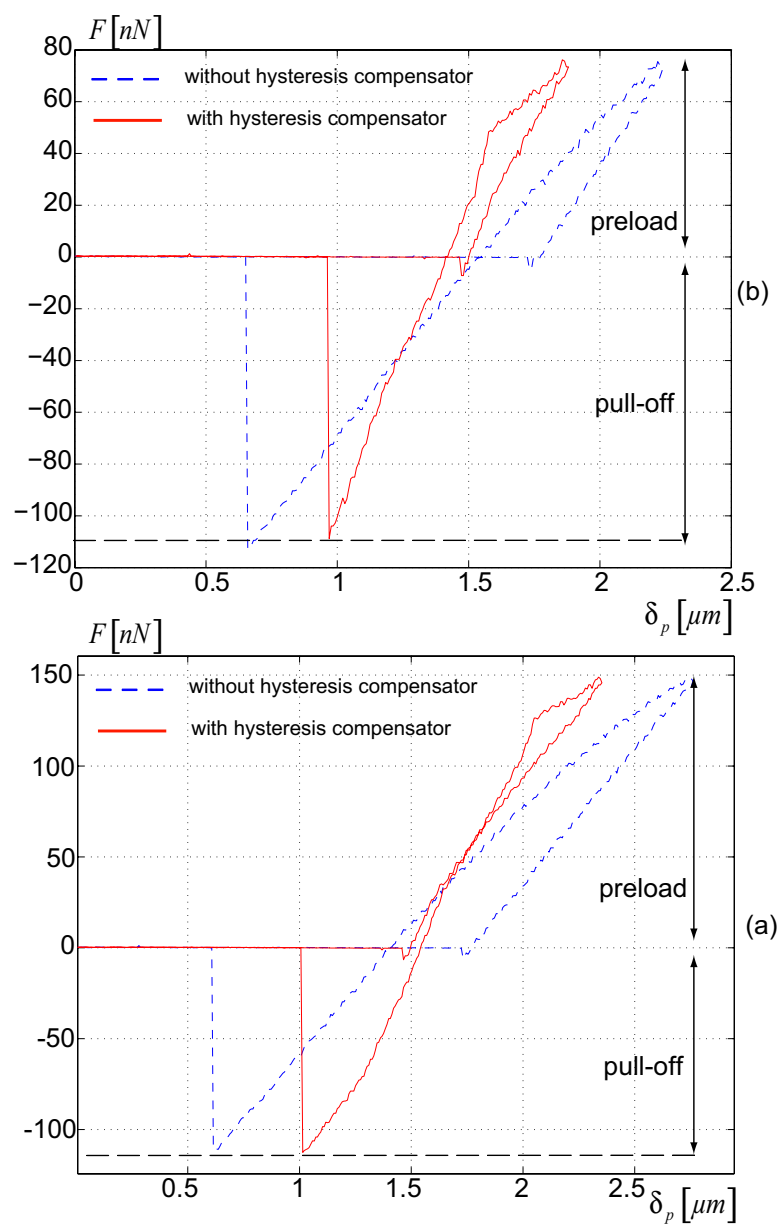

Fig. 11. Experimental result: measurement of the pull-off force between the AFM-lever and a gold substrate.

[8] M. Rakotondrabe, C. Clévy and P. Lutz, "Complete open loop control of hysteretic, creepd and oscillating piezoelectric cantilevers", IEEE Transactions on Automation Science and Engineering, accepted 2009.

[9] K. Kuhnen and H. Janocha, 'Complex hysteresis model of a broad class of hysteretic nonlinearities', Conference on New Actuators, 2002.

[10] A. Falvo, F. Furgiuele and C. Maletta, "Two-way shape memory effect of a $\mathrm{Ti}$ rich NiTi alloy: experimental measurements and numerical simulations", IOP Smart Materials Structures, pp.771-778, 2007.

[11] K. Kuhnen and H. Janocha, "Inverse feedforwrad controller for complex hysteretic nonlinearities in smart-materials systems".

[12] Micky Rakotondrabe, Yassine Haddab and Philippe Lutz, "Quadrilateral modelling and robust control of a nonlinear piezoelectric cantilever", IEEE - Transactions on Control Systems Technology, 2009. 